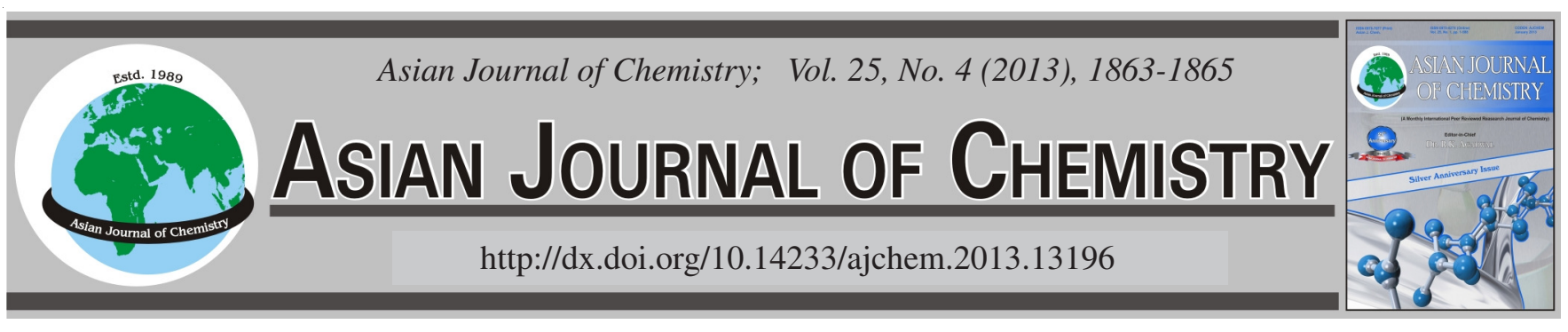

\title{
Growth, Structural, Spectral, Optical and Mechanical Studies of Calcium Mixed Strontium Tartrate Single Crystals
}

\author{
M. Mary Freeda ${ }^{1, *}$, T.H. FreedA ${ }^{2}$ and S. MARY Delphine ${ }^{1}$
}

${ }^{1}$ Department of Physics, Holy Cross College, Nagercoil-629 004, India

${ }^{2}$ Physics Research Centre, S.T. Hindu College, Nagercoil-629 002, India

*Corresponding author: E-mail: maryfreeda13@gmail.com

(Received: 7 December 2011;

Accepted: 5 October 2012)

AJC-12237

\begin{abstract}
Calcium mixed strontium tartrate crystals were grown by controlled diffusion in silica gel at ambient temperature. Structural characterization of the grown crystals was carried out by single crystal and powder X-ray diffraction analysis. The functional groups present in the crystals were identified using Fourier transform infrared spectral analysis. The chemical composition of the sample was verified using EDAX spectrum. The nonlinear optical property of the grown crystal was confirmed by Kurtz-Perry powder technique. The UV-VIS-NIR transmission spectrum was recorded to study the optical transparency of the grown crystal. Mechanical strength of the grown crystals was analyzed by Vicker's microhardness test.
\end{abstract}

Key Words: Gel growth, Characterization, Nonlinear optical materials, X-Ray diffraction, FT-IR, Microhardness.

\section{INTRODUCTION}

Metal tartrates form an important class of materials ${ }^{1}$ as they exhibit interesting electrical and optical properties. Tartrate single crystals show many interesting physical properties such as ferroelectric, piezoelectric, dielectric, optical and other pertinent characteristics ${ }^{2-5}$. In view of these potential characteristics tartrate crystals are exploited for their use in transducers and in several linear and nonlinear mechanical devices. They are also used in nonlinear optical devices, optical transmission characteristics, fabrication of crystal oscillators and controlled laser emission ${ }^{6-8}$.

Among the several tartrate compounds, strontium tartrate and calcium tartrate have received greater attention on account of their ferroelectric, non linear optical and spectral characteristics ${ }^{2,9,10}$. Calcium tartrate crystals are well known for its ferroelectric properties in pure ${ }^{2}$ as well as doped forms ${ }^{11}$ and their structural characterization ${ }^{12}$ and FT-IR and thermal properties ${ }^{13}$ have been reported. Strontium tartrate is an important member of the tartrate family with device applications ${ }^{14}$. Growth and characterization of strontium tartrate single crystals, both pure and doped have been reported by many researchers ${ }^{15-18}$. Growth of mixed crystals of various compositions is a fast growing field of research because of the great need of such crystals for several applications. Recent focus is on tartrate mixed crystals which constitute a very interesting system for academic and research studies. The physico-chemical characterization of calcium strontium tartrate crystals grown from light irradiated sodium meta silicate gel medium was reported $^{19}$. In the present work, we report the growth of calcium mixed strontium tartrate single crystals by gel method and the structural, optical, mechanical and spectral characterization of the grown crystals by single crystal and powder XRD studies, FTIR, optical absorption studies, SHG efficiency measurement, Energy dispersive analysis and microhardness studies.

\section{EXPERIMENTAL}

Calcium mixed strontium tartrate (SCT) crystals were grown in silica gel by employing the test tube diffusion method $^{20}$. Silica gel was prepared by adding sodium meta silicate solution of specific gravity 1.04 to tartaric acid (1 M) drop by drop with continuous stirring with a view to avoid excessive local ion concentration which may cause premature local gelling and make the final solution inhomogeneous and turbid. The $\mathrm{pH}$ of the gel was adjusted to attain the value of 4.0. This gelling mixture was allowed to set in glass tubes of length $20 \mathrm{~cm}$ and diameter $2.5 \mathrm{~cm}$. The gel was found to set in about 4 days. After ensuring firm gel setting, a mixture of aqueous solutions of strontium chloride $(0.7 \mathrm{M})$ and calcium chloride $(0.3 \mathrm{M})$ was poured above the set gel with the help of a pipette, so as to allow the solution to fall steadily along the wall of the tube to prevent the gelled surface from cracking. The supernatant solution diffuses into the gel column and reacts 
with the inner reactant, giving rise to the formation of calcium mixed strontium tartrate crystals. The crystals were harvested after a month. The maximum size of the crystals grown was about $6 \mathrm{~mm} \times 5 \mathrm{~mm} \times 3 \mathrm{~mm}$. All the chemicals used were of AnalaR grade.

\section{RESULTS AND DISCUSSION}

Single crystal X-ray diffraction analysis: Single crystal $\mathrm{X}$-ray diffraction analysis has been carried out to confirm the crystallinity and to find the lattice parameters of the grown crystals. Single crystals of calcium mixed strontium tartrate were subjected to single crystal X-ray diffraction studies using BRUKER NONIUS CAD4 single crystal X-ray diffractometer to obtain the unit cell parameters. The obtained results show that the grown crystals belong to orthorhombic system with space group $\mathrm{P} 22_{1} 2_{1}$. The determined lattice parameters are a $=9.176 \AA, \mathrm{b}=9.579 \AA, \mathrm{c}=10.505 \AA, \mathrm{V}=923.6 \AA^{3}$ and $\alpha=\beta$ $=\gamma=90^{\circ}$.

Powder X-ray diffraction analysis: The grown SCT crystals were subjected to powder X-ray diffraction studies. The X-ray powder diffraction pattern of the crystals was obtained using XPERT-PRO X-ray diffractometer with $\mathrm{CuK}_{\alpha}$ radiation of wavelength $1.54056 \AA$ operating at a voltage of $40 \mathrm{kV}$ and a current of $20 \mathrm{~mA}$. The scanning rate was maintained at $1.6^{\circ} / \mathrm{min}$ over a $2 \theta$ range of $10-70^{\circ}$ employing the reflection mode for scanning. Well defined peaks at specific $2 \theta$ values show high crystallinity of the grown crystals. All the reflections of the powder XRD pattern were indexed using the INDEXING and TREOR software packages. The indexed powder XRD diffractogram of SCT crystals is shown in Fig. 1. From the XRD analysis it is observed that the grown crystals belong to orthorhombic system with space group $\mathrm{P} 22_{1} 2_{1} 2_{1}$. The lattice parameters obtained from the indexed XRD pattern using the UNIT CELL software package are $\mathrm{a}=9.1931 \AA, \mathrm{b}=$ $9.5693 \AA, c=10.5061 \AA, V=924.2453 \AA^{3}$ and are observed to be comparable with the values obtained from single crystal XRD studies.

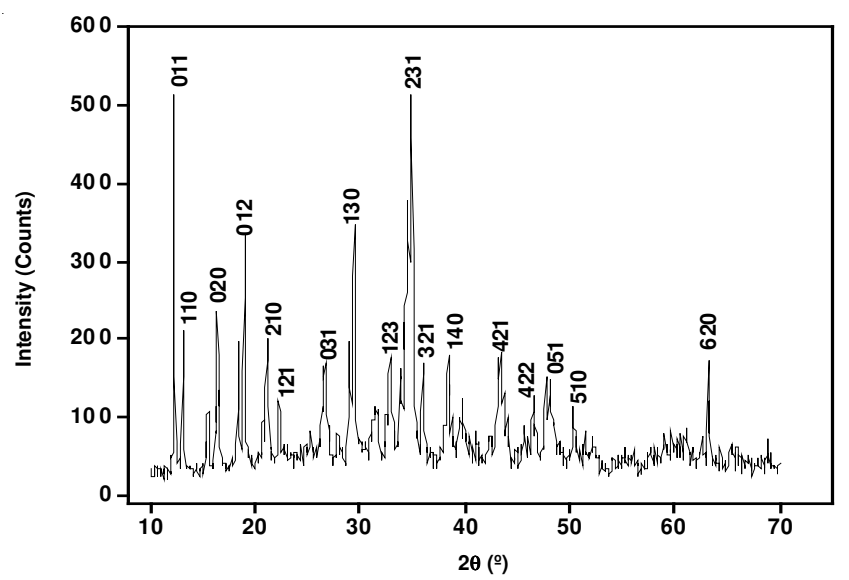

Fig. 1. Powder XRD pattern of SCT crystals

Fourier transform infrared analysis: The FTIR analysis is a technique that provides information about the chemical bonding or molecular structure of materials. The FTIR spectrum of the grown crystals was recorded in the wave number range 4000-400 $\mathrm{cm}^{-1}$ using a Perkin- Elmer Fourier transform infrared spectrometer (Model: Spectrum RXI) using KBr pellet technique. In the FTIR spectrum, the absorption bands in the region $3577-2599 \mathrm{~cm}^{-1}$ are due to $\mathrm{OH}$ stretching mode, water and $\mathrm{C}-\mathrm{H}$ stretch. The band at $1590 \mathrm{~cm}^{-1}$ is attributed to the $\mathrm{C}=\mathrm{O}$ stretch of carbonyl group. The peak at $1388 \mathrm{~cm}^{-1}$ is assigned to $\mathrm{C}-\mathrm{OH}$ in bending mode. The peak at $1326 \mathrm{~cm}^{-1}$ is due to -OCH stretching mode. The peaks at 1283 and 1146 $\mathrm{cm}^{-1}$ are attributed to C-O stretching mode. The peaks at 1062 and $961 \mathrm{~cm}^{-1}$ are assigned to $\mathrm{C}-\mathrm{O}$ stretching and C-C stretching modes. The peak at $712 \mathrm{~cm}^{-1}$ is due to the deformation vibrations of $\mathrm{CO}_{2}$. The peak at $533 \mathrm{~cm}^{-1}$ is attributed to metal-oxygen bond. The presence of $\mathrm{O}-\mathrm{H}$ bond, $\mathrm{C}-\mathrm{O}$ bond, water of hydration and carbonyl $\mathrm{C}=\mathrm{O}$ bond are established from the spectra.

Optical absorption spectral analysis: The optical absorption spectrum of the grown SCT crystal was recorded in the wavelength range $200-900 \mathrm{~nm}$ using VARIAN CARY $5 \mathrm{E}$ spectrophotometer. It is inferred from the spectrum that the grown crystals have low absorbance in the entire visible and NIR regions and show maximum absorption at UV region. This is one of the most desirable properties of the crystals for the device fabrication.

Second harmonic generation (SHG) test: Kurtz and Perry powder technique ${ }^{21}$ is extremely useful for the initial testing of materials for second harmonic generation (SHG). The non-linear optical property of the grown crystal was tested by Kurtz-Perry Powder technique. The crystal was grounded into a homogenous powder and densely packed between two transparent glass slides. A Q switched Nd:YAG laser beam of wavelength $1064 \mathrm{~nm}$ with an input power of $0.68 \mathrm{~J} /$ pulse, pulse width of $6 \mathrm{~ns}$ and repetition rate of $10 \mathrm{~Hz}$ was directed on the sample. The doubling of frequency was confirmed by the emission of green radiation of wavelength $532 \mathrm{~nm}$ using a photomultiplier tube. A sample of powdered potassium dihydrogen phosphate (KDP) was used as the reference material in the SHG measurement. From the obtained data, the efficiency of second harmonic generation was found to be 0.4 times that of the standard KDP.

Energy dispersive X-ray analysis: In order to confirm the presence of the elements in the grown SCT crystals, the sample of grown crystals were subjected to Energy Dispersive X-ray analysis. The grown SCT crystal was analyzed using an energy dispersive X-ray spectrometer (JEOL Model JED2300). The obtained spectrum (Fig. 2) confirms the presence of strontium and calcium.

Vicker's microhardness test: Hardness is one of the important mechanical properties of a solid material. Microhardness studies of any system has direct correlation with the crystal structure and is very sensitive to the presence of any other phase or phase transition and temperature conditions prevalent in the system. The grown CST crystals were tested for their mechanical property using Shimadzu HMV-2 Vicker's microhardness tester fitted with a diamond indenter. Indentations were made for various loads from 25-100 g. Several trials of indentation were carried out and the average diagonal lengths were measured for an indentation time of $5 \mathrm{~s}$. The Vicker's hardness number $\left(\mathrm{H}_{v}\right)$ was calculated from the relation

$$
\mathrm{H}_{\mathrm{v}}=1.8544 \frac{\mathrm{P}}{\mathrm{d}^{2}} \mathrm{Kg} / \mathrm{mm}^{2}
$$




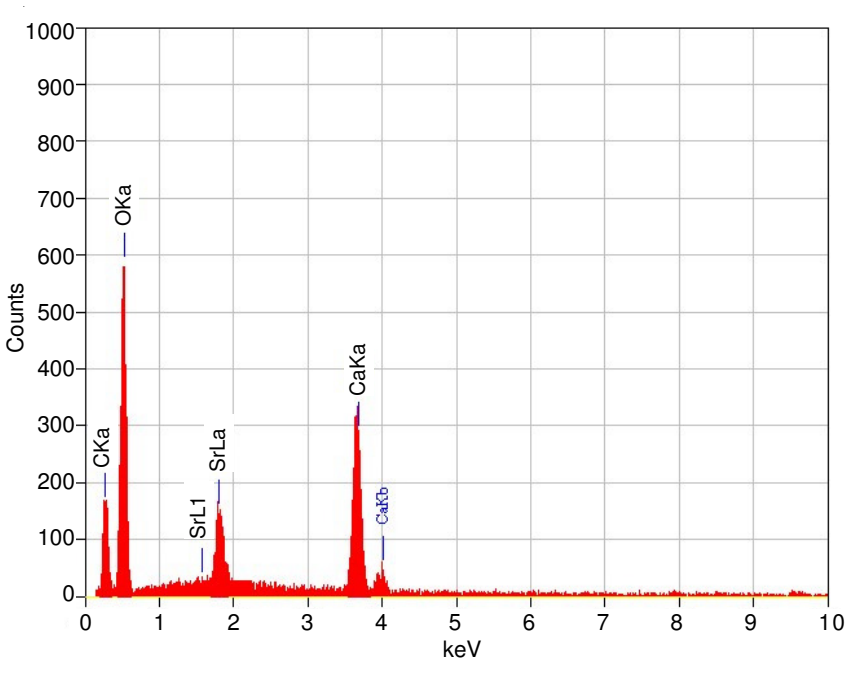

Fig. 2. EDAX spectrum of SCT crystal

where $\mathrm{P}$ is the load in $\mathrm{Kg}$, $\mathrm{d}$ the diagonal length of the indentation impression in $\mathrm{mm}^{22}$.

The values of $\mathrm{H}_{v}$ were calculated using (1) for various loads. It was observed that the hardness increases with increase in load and above $100 \mathrm{~g}$, cracks develop on the smooth surface of the crystal due to release of internal stresses generated locally by indentation ${ }^{23}$. This can be explained qualitatively on the basis of the depth of penetration of the indenter ${ }^{24}$. For small loads only a few surface layers are penetrated by the indenter. The measured hardness is characteristic of these layers and $\mathrm{H}_{\mathrm{v}}$ increases with load in this region. With increase in load, the overall effect is due to the surface as well as the inner layer of the sample. The work hardening coefficient $n$ was determined from the slope of $\log \mathrm{P}$ versus $\log \mathrm{d}$ plot using least square fit method. The value of $n$ was found to be $>2$. According to Onitsch ${ }^{25}$, if $n>2$, the materials are said to be soft ones. He also found that microhardness increases with increasing load when $n>2$ and decreases with increasing load when $\mathrm{n}<2$. The increase in $\mathrm{H}_{\mathrm{v}}$ for increasing load observed in the present study is in good agreement with the theoretical prediction.

\section{Conclusion}

Good quality single crystals of calcium mixed strontium tartrate were grown in silica gel. Structural characterization of the grown crystals was carried out by single crystal and powder X-ray diffraction studies and the lattice parameters have been evaluated. The FTIR spectrum of the SCT crystals reveals the presence of various functional groups present in the crystal. The optical studies confirm that the grown crystal is transparent in the entire visible region. The powder SHG test shows that the conversion efficiency is 0.4 times as that of KDP. Qualitative elemental analysis (EDAX) confirms the presence of strontium and calcium. Vicker's microhardness test shows that the crystals grown in the present study fall in the category of soft materials.

\section{ACKNOWLEDGEMENTS}

One of the authors (M. Mary Freeda) is thankful to the UGC, New Delhi for awarding Teacher Fellowship under FDP during XI plan period. The support extended by RRL (Trivandrum), Crecent Engineering College (Chennai), Loyola College (Chennai), St. Joseph's College (Trichy) and M.K. University ( Madurai) are gratefully acknowledged.

\section{REFERENCES}

1. G.A. Kiosse, In ed.: T.I. Malinowskii, In Crystal Structure of Inorganic Compounds, (Shtuntsa Press, Kishineve), p. 103 (1974).

2. H.B. Gon, J. Cryst. Growth, 102, 501 (1990).

3. S.K. Arora, V. Patel, B. Amin and A. Kothari, Bull. Mater. Sci., 27, 141 (2004).

4. V.S. Yadava and V.M. Padmanabhan, Acta Cryst. B, 29, 493 (1973).

5. C.C. Desai and A.H. Patel, Bull. Mater. Sci., 11, 31 (1988).

6. M.V. Hobden, J. Appl. Phys., 38, 4365 (1967).

7. S.S. Sekarapandian, K. Vivekanandan and P. Kolandaivel, Cryst. Res. Technol., 34, 873 (1996).

8. S.K. Arora, V. Patel, A. Kothari and B. Chudasama, Mater. Sci. Eng. B, 113, 263 (2004).

9. C. Medrano, P. Gunter and H. Arend, Phys. Status Solidi, 14B, 749 (1987).

10. P. Selvarajan, B.N. Das, H.B. Gon and K.V. Rao, J. Mater. Sci. Lett., 12, 1210 (1993).

11. S.R. Suthar and M.J. Joshi, Cryst. Res. Technol., 41, 664 (2006).

12. M.E. Torres, T. Lopez, J. Stockel, X. Solans, M. Garcia-Valles, E. RodriguezCastellon and C. Gonzalez-Silingo, J. Solid State Chem., 163, 491 (2002).

13. X. Sahaya Shajan and C. Mahadevan, Cryst. Res. Technol., 40, 598 (2005).

14. M.H. Rahimkutty, K.R. Babu, K.S. Pillai, M.R.S. Kumar and C.M.K. Nair, Bull. Mater. Sci., 24, 249 (2001).

15. S.K. Arora, V. Patel, B. Chudasama and B. Amin, J. Cryst. Growth, 275, e657 (2005).

16. A. Firdous, I. Quasim, M.M. Ahmad and P.N. Kotru, Cryst. Res. Technol., 43, 1015 (2008).

17. F.J. Rethinam, D. Arivuoli, S. Ramasamy and P. Ramasamy, Cryst. Res. Technol., 28, 861 (1993).

18. B. Gowri and X.S. Shajan, Mater. Lett., 60, 1338 (2006).

19. K. Suryanarayana and S.M. Dharmaprakash, J. Phys. Chem. Solids, 58, 1599 (1997).

20. H.K. Henisch, Crystal Growth in Gels, Dover Publications Inc., New York, p. 20 (1996).

21. S.K. Kurtz and T.T. Perry, J. Appl. Phys., 39, 3798 (1968).

22. K.K. Rao, V. Surender and B.S. Rani, Bull. Mater. Sci., 25, 641 (2002).

23. T. Balakrishnan and K. Ramamurthi, Mater. Lett., 62, 65 (2008).

24. J.B. Charles and F.D. Gnanam, J. Mater. Sci. Lett., 12, 1395 (1993).

25. E.M. Onitsch, Mikroskopie, 2, 131 (1947). 